EISSN: 2707-0425

East African Journal of Science, Technology and Innovation, Vol. 3 (1): December 2021

This article is licensed under a Creative Commons license, Attribution 4.0 International (CC BY NC SA 4.0)

\title{
Molecular detection of Campylobacter species from human and cattle faecal samples in Kilosa District, Tanzania
}

\author{
1,5*GAHAMANYI N., 1MBOERA L E G., ${ }^{2}$ MATE M I., ${ }^{3}$ MUTANGANA D., 4 AMACHAWADI R \\ G., ${ }^{5}$ YOON K Y., 1,5MABWI H A., ${ }^{5} \mathrm{CHA}$ K H., 5PAN C H., 1 KOMBA E V G
}

1SACIDS Foundation for One Health, College of Veterinary Medicine and Biomedical Sciences, Sokoine University of Agriculture, P.O. Box 3015, Chuo Kikuu, Morogoro, Tanzania;

${ }^{2}$ School of Medicine, Muhimbili University of Health and Allied Sciences, P.O. Box 65001, Dar es Salaam, Tanzania;

${ }^{3}$ College of Science and Technology, University of Rwanda, P.O. Box 3900, Kigali, Rwanda;

${ }^{4}$ Department of Clinical Sciences, College of Veterinary Medicine, Kansas State University, Manhattan, Kansas, 66506-5606, United States of America;

${ }^{5}$ Natural Product Informatics Research Center, KIST Gangneung Institute of Natural Products, Gangneung 25451, Republic of Korea ${ }^{6}$ Division of Bio-Medical Science and Technology, KIST School, Korea University of Science and Technology, Seoul 02792, Republic of Korea

*Corresponding author: noel.gahamanyi@kist.re.kr

\section{Abstract}

A growing number of Campylobacter species other than C. jejuni and C. coli have been considered as emerging human and animal pathogens but their contribution to human gastroenteritis is poorly documented. This study aimed at detecting Campylobacter species from human and cattle faecal samples in Kilosa District, Tanzania using molecular techniques without culture. Seventy (70) faecal samples were collected from five diarrheic and 65 non-diarrheic human patients attending Kilosa District Hospital in Tanzania from July to October 2019. During the same period, 30 faecal samples were also collected from healthy cattle in the same district. Genus and species identification of Campylobacter was conducted on the samples using molecular techniques [the polymerase chain reaction (PCR) and 16S rRNA sequencing]. Phylogenetic analysis was carried out by comparison of the $16 \mathrm{~S}$ rRNA gene sequences to reference strains by the Neighbor-Joining method in MEGA X. Campylobacter species detection rate by PCR was $65.7 \%$ $(46 / 70)$ and $20 \%(6 / 30)$ in humans and cattle, respectively. There were five human diarrheic cases, four of which were positive for Campylobacter and of these, two were children $\leq 15$ years of age. In humans, 16S rRNA sequencing revealed that $C$. concisus was the most predominant species occurring at a frequency of $37.8 \%$ (14/37), followed by uncultured Campylobacter spp. $24.3 \%$ (9/37) and C. hominis $21.6 \%$ (8/37). The least represented species were C. jejuni and C. lanienae, all occurring at $2.7 \%$ (1/37). In cattle, five (100\%) sequenced PCR products matched with C. lanienae. Phylogenetic analysis revealed that with the exception of C. lanienae, $16 \mathrm{~S}$ rRNA sequences of Campylobacter species were closely related to the reference strains used (Percent identity: 90.51-96.56\%). Based on our findings, we recommend that molecular techniques, mainly PCR be adopted for the direct detection of Campylobacter species during laboratory screening and surveillance studies.

Keywords: Campylobacter, molecular diagnosis, polymerase chain reaction, sequencing, gastroenteritis, humans, cattle, Tanzania

Received: $\quad 04 / 08 / 21$

Accepted: $\quad 22 / 11 / 21$

Published: $\quad$ 17/12/21 
Cite as: Gahamanyi et al., (2021) Molecular detection of Campylobacter species from human and cattle faecal samples in Kilosa district, Tanzania. East African Journal of Science, Technology and Innovation 2(4).

\section{Introduction}

Campylobacter species, one of the zoonotic pathogens causing gastroenteritis, are responsible for 96 million cases of diarrhoea in humans each year (Ruiz-Palacios, 2007; Havelaar et al., 2013). Campylobacter has also been reported to cause infertility in cattle and abortions in sheep, goats, and cattle (Sahin et al., 2017). Poultry, mainly chickens, are considered as the primary reservoir of Campylobacter but various domestic and wild animals have also been reported as potential sources of Campylobacter (Gahamanyi et al., 2021). Apart from the animal reservoirs, it is likely that the natural environment (soil and water) plays a key role in transmission, either directly to humans or indirectly via farm animals (Bronowski et al., 2014). The incidence of human cases of campylobacteriosis has been increasing in both developed and developing countries throughout the world (Kaakoush et al., 2015; Sulaiman et al., 2020). However, Campylobacter infections have been considered as hyperendemic in most of the low and middle-income countries (LMICs) (Coker et al., 2002) and the major risk factors include eating contaminated poultry products or drinking unboiled water (Kaakoush et al., 2015). In Africa, the prevalence of human campylobacteriosis varies from $7.7-18.5 \%$, and Campylobacter is persistently found in stools of both diarrheic and non-diarrheic children (Gahamanyi et al., 2020). This is often linked to poor hygiene and sanitation (Osbjer et al., 2016). However, there is a limited number of reports on the prevalence of Campylobacter both in humans and animals probably because Campylobacter is not among the pathogens screened for by medical and veterinary hospitals in most of the developing countries. The occurrence of Campylobacter in Tanzania may be higher than what is reported due to the lack of enough reporting and antibiotic prescription is done before laboratory confirmation (Rukambile et al., 2021). In some rural areas, people share the same house with livestock and the likelihood of contaminating kitchen utensils, environment, and playground with feces is high, and thus increasing the risk of contracting zoonotic infections (Rukambile et al., 2021).

Apart from human gastroenteritis, Campylobacter infections have been associated with several complications such as the reactive arthritis (RA), Reiter's Syndrome (RS), irritable bowel syndrome (IBS), Guillain-Barré Syndrome (GBS), Inflammatory Bowel Disease (IBD), Crohn's disease (CD), and Ulcerative Colitis (UC) (Esan et al., 2017).

It is known that the majority of human cases of campylobacteriosis are self-limiting and do not require antibiotic treatment (Guévremont et al., 2006). However, treatment is advised for severe cases of Campylobacter infections especially among children and immune-compromised individuals (Kaakoush et al., 2015). Drugs of choice include erythromycin (macrolide) and ciprofloxacin (quinolone) but Campylobacter strains that are resistant to these drugs are increasingly being reported (Sproston et al., 2018). The increasing resistance is partly attributed to misuse of antimicrobials in both human and veterinary medicine (Guévremont et al., 2006).

Campylobacter jejuni and C. coli account for more than $90 \%$ of human campylobacteriosis cases (Moore et al., 2005). However, atypical Campylobacter species are gaining considerable attention as important human and animal pathogens (Zhang et al., 2009; Man, 2011). Infections caused by Campylobacter are usually under-reported due to difficulties in isolation procedures (Lastovica, 2016). For instance, it has been estimated that $40 \%$ of the bacteria from human faeces diagnosed through microscopy cannot be cultured in the laboratory (Berg, 1996). In contrast to other gastrointestinal pathogens, the culturing of Campylobacter species is laborious due to their microaerophilic nature and vulnerability to temperature fluctuations (Park, 2002). Conventional procedures for the isolation 
and identification of Campylobacter from various sources require bacterial enrichment for two days and subculturing to selective agar, followed by phenotypic identification (Jacob et al., 2011). Furthermore, commonly used selective media and added antimicrobials may inhibit the growth of certain Campylobacter species (Bessede et al., 2011). Species like C. concisus, C. sputorum, C. curous, C. rectus, and some strains of C. hyointestinalis grow in a microaerobic atmosphere that is supplemented with hydrogen to facilitate their recovery (Jacob et al., 2011). Moreover, Campylobacter species may become dormant as viable but non-culturable (VBNC) forms difficult to grow on commonly used media (Singh et al., 2011) unless a primary enrichment step is included in the isolation protocol (Thornval and Hoorfar, 2021). Consequently, the epidemiology and role of non-C. jejuni/C. coli species in human gastroenteritis are not fully understood ( Lastovica, 2006).

The emerging Campylobacter species have been neglected but the integration of molecular techniques and suitable culture media in current diagnostic tests has helped in promoting the awareness of atypical species as relevant human and animal pathogens (Lastovica, 2016). Campylobacter concisus has been associated with gastroenteritis, inflammatory bowel disease, gingivitis, and periodontitis (Man, 2011; Liu et al., 2018). Campylobacter hominis has been isolated in a blood sample of a septicemic patient (Linscott $e t$ al., 2005), while C. gracilis has been associated with bacteremia, head infections, periodontitis and empyema (Gorkiewicz et al., 2003; Liu et al., 2018). In cattle, the most commonly reported species are C. fetus, C. lanienae, C. sputorum, C. jejuni, and C. hyointestinalis ( Linton et al., 1997; Inglis and Kalischuk, 2003; Mshelia et al., 2010).

The polymerase chain reaction (PCR) and other molecular diagnostic tests based on nucleic acids are attractive due to their benefits including their higher sensitivity, ease-of-use, improved turnaround time, relatively low cost, and potential to be fully automated (Ghosh et al., 2014). The breakthrough in technology and easy access to commercial kits has led to shifting from traditional laboratory diagnostic techniques to the newer molecular ones (Amjad, 2020). The analysis of the $16 \mathrm{~S}$ rRNA gene by PCR and sequencing techniques has assisted in the phylogenetic identification of Campylobacter species including those unidentified by conventional techniques (Al-Nasrawi, 2016). Some of the disadvantages of molecular-based techniques include expensive initial setups, lack of discrimination between living and dead bacteria, and not contributing to public health surveillance and outbreak investigations due to lack of isolates (Amjad, 2020).

Despite being time-consuming (up to five days to get pure colonies), Campylobacter isolation by culture is still useful as it allows to get pure colonies and testing of antimicrobial susceptibility (Ghosh et al., 2014). Bacterial isolates are crucial for understanding and evaluating phenotypic and genotypic characteristics of individual isolates but they can also be used for surveillance studies by national or international organizations (McLain et al., 2016).

In Tanzania and most of the low and middleincome countries (LMICs), the reports on the role of Campylobacter spp. in gastroenteritis are scanty due to limited capacity in laboratory diagnosis and the absence of national surveillance programmes (Coker et al., 2002; Gahamanyi et al., 2020). Thus, the information available for both human and animal campylobacteriosis is limited (Komba et al., 2013) which undermines its importance as a public health concern. The current study aimed at molecular detection of Campylobacter species in human and cattle faecal samples in Kilosa District, Tanzania using PCR amplification of the 16S rRNA gene and Sanger sequencing.

\section{Materials and methods}

\section{Study design and sample collection}

This cross-sectional study was conducted in Kilosa district of east-central Tanzania $\left(6^{\circ} \mathrm{S}\right.$ and $8^{\circ} \mathrm{S}$, and between $36^{\circ} 30^{\prime}$ and $38^{\circ} \mathrm{E}$ ) from July 2019 to October 2019. Human stool samples were randomly obtained from patients with abdominal discomfort seeking medical care at Kilosa District Hospital during the time of the study. We selected the $1^{\text {st }}, 3^{\text {rd }}, 5^{\text {th }}$, etc. patients reporting to the Microbiology Laboratory for stool examination. Cattle faecal samples were 
randomly collected using sterile gloves from healthy lactating cows belonging to Mbumi ward. The ward was purposively selected based on the number of lactating cows and accessibility. Farms and cows were randomly selected by lottery. Identified farms were given numbers and selection was done by the lottery through picking the corresponding numbers. Upon reaching each farm, animals were also given numbers and the same procedure was repeated. The criteria to include the animals were being a lactating cow and apparently being healthy.

A total of 70 human stool samples and 30 cattle rectal grab faecal samples were collected in sterile dry screw-top containers containing Dimethyl Sulfoxide (DMSO), packed in a cool box, and transported to the Microbiology Laboratory at Sokoine University of Agriculture for DNA extraction within 8 hours of collection. In case DNA extraction could not be done within 8 hours of sample collection, the stool samples were refrigerated at $4{ }^{\circ} \mathrm{C}$ and processed the following day.

\section{DNA extraction and Campylobacter species identification}

Approximately, $1 \mathrm{~g}$ faecal sample in DMSO was diluted $(10 \% \mathrm{wt} / \mathrm{vol})$ in buffered peptone water (BPW) $(9 \mathrm{ml})$ and vortexed until the sample was thoroughly homogenized. Two hundred (200) $\mu \mathrm{L}$ of the homogenized faecal sample was used for genomic DNA extraction using Quick-DNA ${ }^{\mathrm{TM}}$ Faecal/Soil Microbe Microprep Kit (Zymo Research Corp, Irvine, California, USA) based on the manufacturer's instructions. Eluted DNA concentration and purity were checked using a NanoDrop ${ }^{\mathrm{TM}}$ spectrophotometer (Biochrom, Cambridge, England) before storage at $-20^{\circ} \mathrm{C}$.

Detection of Campylobacter was done by multiplex PCR using specific primers cj0414 for C. jejuni and ask for C. coli as previously described (YamazakiMatsune et al., 2007). Then, PCR of the 16S rRNA gene was performed on DNA samples negative for $C$. jejuni and $C$. coli using genus-specific primers including a 19bp-forward primer $(\mathrm{C} 412 \mathrm{~F})$ and an 18bp-reverse primer with complementary sequence (C1228R) as previously described (Linton et al., 1996). Positive control DNA was extracted from Campylobacter jejuni (ATCC $^{\circledR}$ $33560^{\mathrm{TM}}$ ) while deionized water was included as the negative control. The PCR final volume was $25 \mu \mathrm{L}$, including $12.5 \mu \mathrm{L}$ of $2 \mathrm{X}$ Master Mix (Thermo Fisher Scientific, Seoul, South Korea), 1 $\mu \mathrm{L}(10 \mu \mathrm{M})$ of $\mathrm{C} 412 \mathrm{~F}$ primer, $1 \mu \mathrm{L}(10 \mu \mathrm{M})$ of C1228R primer, $1 \mu \mathrm{L}$ of template DNA, and $9.5 \mu \mathrm{L}$ of sterile deionized water. All primers were made by Integrated DNA Technologies, Inc. (Singapore Science Park, Singapore).

The DNA amplification was performed using the model MiniAmp ${ }^{\mathrm{TM}}$ plus Thermal Cycler (Applied Biosystems, Massachusetts, USA). The cycling conditions used were initial denaturation at $95^{\circ} \mathrm{C}$ for 5 minutes, 35 cycles each of $94^{\circ} \mathrm{C}$ for 30 seconds, $55^{\circ} \mathrm{C}$ for 45 seconds and $72^{\circ} \mathrm{C}$ for 45 seconds, and a final extension at $72^{\circ} \mathrm{C}$ for 7 minutes. The PCR products were held at $4^{\circ} \mathrm{C}$ before analysis. PCR products $(5 \mu \mathrm{l})$ were mixed with $2 \mu 1$ Loading STAR (Dyne bio, Seongnam-si, Korea) diluted with $5 \mu \mathrm{l}$ of nuclease-free water and analyzed by gel electrophoresis where $10 \mu \mathrm{l}$ of the mixture was loaded onto $1.5 \%$ SeaKem ${ }^{\circledR} \mathrm{LE}$ Agarose gel (Lonza Inc.-Rockland, Maine, USA) in 0.5X TAE buffer. After electrophoresis, PCR product bands were visualized using a Dual UV Transilluminator (Core Bio System, Huntington Beach, California, USA) under ultraviolet (UV) light. Then, the image was photographed with iBright $^{\mathrm{TM}}$ CL1000 Imaging System (Thermo Fisher Scientific, Seoul, South Korea). The size of the amplification products (816bp) obtained was compared to the Dyne 100 bp DNA ladder (Dyne bio, Seongnam-si, Korea). The PCR products (816 bp) were purified using Pure Link TM Quick PCR purification Kit (Invitrogen, Vilnius, Lithuania) and sequenced at SolGent (Solutions for Genetic technologies, Daejeon, South Korea) using the Campylobacter genus-specific primers by Sanger method. However, during purification of PCR products, nine samples from humans and one from cattle did not yield enough DNA required by the sequencing company.

\section{Data analysis}

The data were analyzed with GraphPad Prism 8.4.0 (GraphPad Software, La Jolla, California, USA; 2020). Descriptive statistics (frequencies and percentages) were computed to determine proportions for different attributes. The GenBank sequences with the best and the high scoring matches with sequences of this study were selected using the NCBI BLASTN search. 
Sequences were edited, aligned, and analyzed using BioEdit sequence alignment software (version 7.2.6.1) (Hall, 1999). Multiple sequence alignment by Muscle (Edgar, 2004), computation of evolutionary distances by the Jukes-Cantor method (Jukes and Cantor, 1969), and the phylogenetic tree building by the NeighborJoining (NJ) method (Saitou and Nei, 1987) were done with the Molecular Evolutionary Genetics Analysis (MEGA X) software (MEGA Inc, Englewood, New Jersey, USA) (Kumar et al., 2018). The phylogenetic analysis was carried out by comparing the sequences of this study to $16 \mathrm{~S}$ rRNA genes of reference strains downloaded from LPSN (Parte et al., 2020). To confirm the reliability of our analysis, bootstrap analysis was performed with 1,000 resampled datasets and it was taken to represent the evolutionary history of the taxa analyzed (Felsenstein, 1985). All the 16S rRNA gene sequences derived from sequencing were submitted to GenBank for obtaining accession numbers.

\section{Results}

A total of 70 human stool samples (male $=35$; female $=35$ ) were collected. The age of patients ranged from 2 to 89 years with 10 patients $(14.3 \%)$ being children $\leq 15$ years of age. Overall, the detection rate of Campylobacter spp. in human samples was $65.7 \%$. The PCR products with predicted size $(816 \mathrm{bp})$ were obtained in some of the screened samples (Figure 1). Of the Campylobacter spp. positive samples $(\mathrm{n}=46), 24$ $(52.2 \%)$ were from females and $22(47.8 \%)$ were from males. Campylobacter species were detected in nine of the $10(90 \%)$ children $\leq 15$ years of age. Of the five diarrheic cases, four were positive for Campylobacter. Of the diarrheic patients, two were children $\leq 15$ years. Campylobacter species were detected in six $(20 \%)$ of the 30 faecal samples collected from cattle.

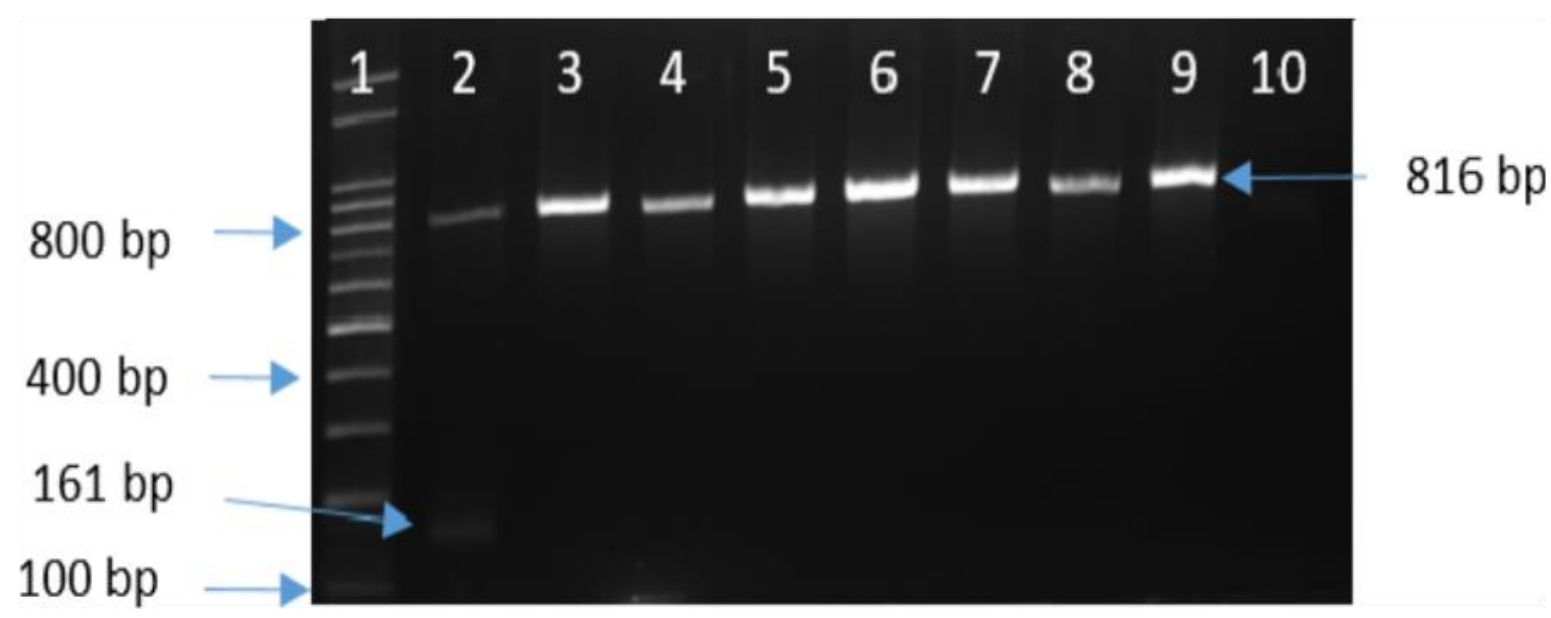

Figure 1. PCR products showing bands for Campylobacter genus (816 bp) and Campylobacter jejuni (161 bp). Lanes: 1: 100bp molecular weight marker; 2-4: bands from human samples; 5: positive control; 6-9: bands from cattle samples; 10: negative control. 


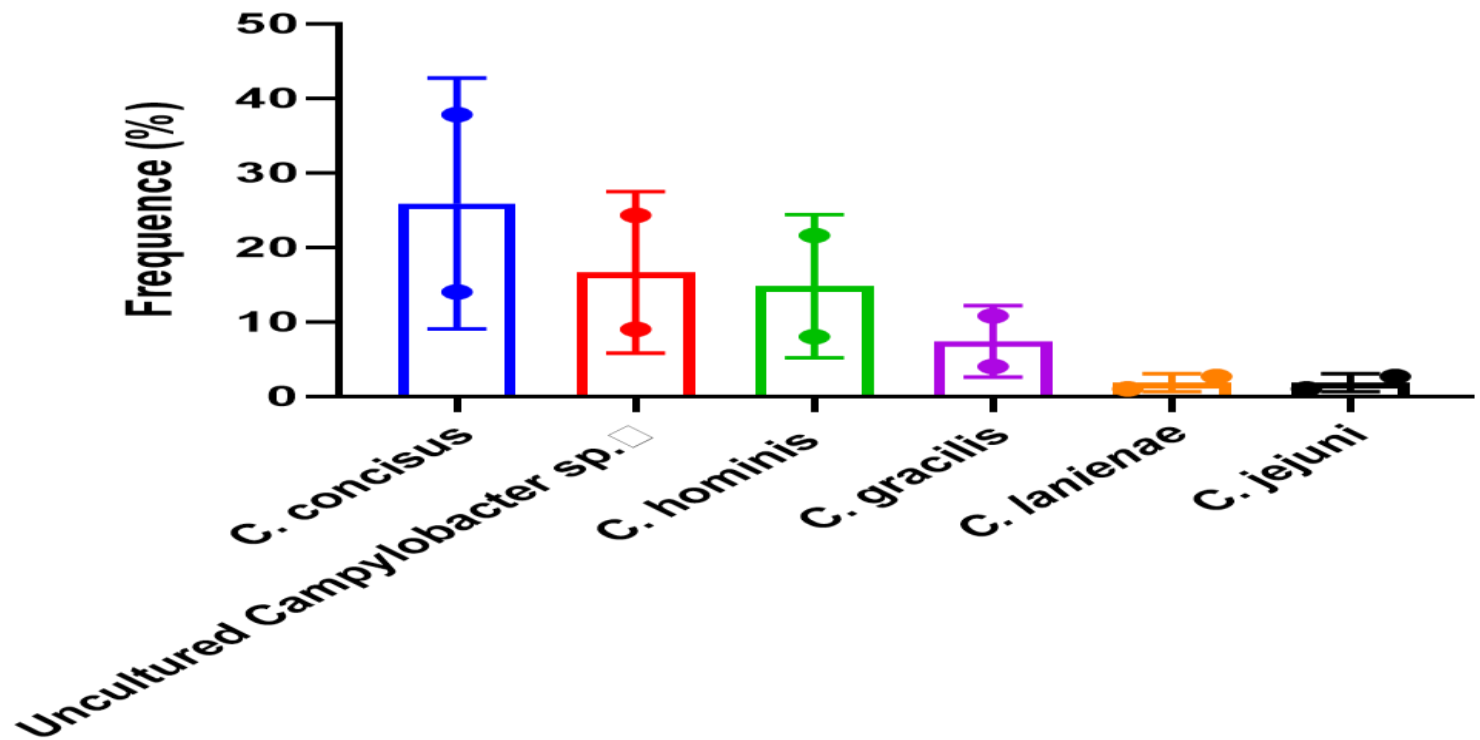

Species

Figure 2. Distribution of Campylobacter species from human samples identified by sequencing. The dots on the whiskers of the box plot (outside the box) refer to the detection rates of each species in percentage while the dots inside the box refer to the frequency of each species

The results of sequencing confirmed the presence of Campylobacter species in all submitted sequences ( 37 from humans and 5 from cattle). The remaining PCR products did not give enough quantity of DNA required by the sequencing company after the purification step. The species were confirmed based on percent identity (above $99 \%)$, the query cover, and the E-value. In humans, C. concisus was the most prevalent $(37.8 \%)$, followed by uncultured Campylobacter spp. $(24.3 \%)$, C. hominis $(21.6 \%)$, and C. gracilis (10.8\%). Campylobacter lanienae and C. jejuni occurred at a frequency of $2.7 \%$ each (Figure 2). For cattle, all the five $(100 \%) 16 \mathrm{~S}$ rRNA sequences matched with $C$. lanienae. The percent identity between species was: $C$. concisus and $C$. hominis $(92.91 \%), \quad$ C. concisus and uncultured Campylobacter spp. (91.78\%), C. concisus and C. jejuni $(93.49 \%)$, and C. jejuni and C. hominis $(91.14 \%)$. The pairwise distances between species were: $C$. concisus and $C$. hominis $(0.07), C$. concisus and uncultured Campylobacter spp. (0.08), C. concisus and C. jejuni (0.07), and C. jejuni and C. hominis (0.1). The mean genetic distance was 0.07 .
The $16 \mathrm{~S}$ rRNA genes of Campylobacter spp. from this study were compared with 16S rRNA sequences of different strains of Campylobacter spp. by BLASTN search. Following submission to the GenBank, gene sequences were allocated with the following accession numbers: MT126449 to MT126453; MT130973 to MT130991; and MT131150 to MT131167.

The phylogenetic analysis was carried out by comparing the 16S rRNA genes of this study to $16 \mathrm{~S}$ rRNA genes of reference strains (C. concisus, C. hominis, C. gracilis, and C. lanienae) and uncultured Campylobacter. The analysis of sequence data from Campylobacter species in this study revealed a high nucleotide sequence similarity to different reference strains. Campylobacter hominis clustered closer to C. gracilis than it was with $C$. concisus. It was also noted that $C$. lanienae formed a separate cluster at the bottom of the tree. Uncultured Campylobacter and Campylobacter spp. RM 12175 were also found among the sequences of this study (Figure 3). The tree was rooted using Helicobacter aurati and Arcobacter molluscorum. 


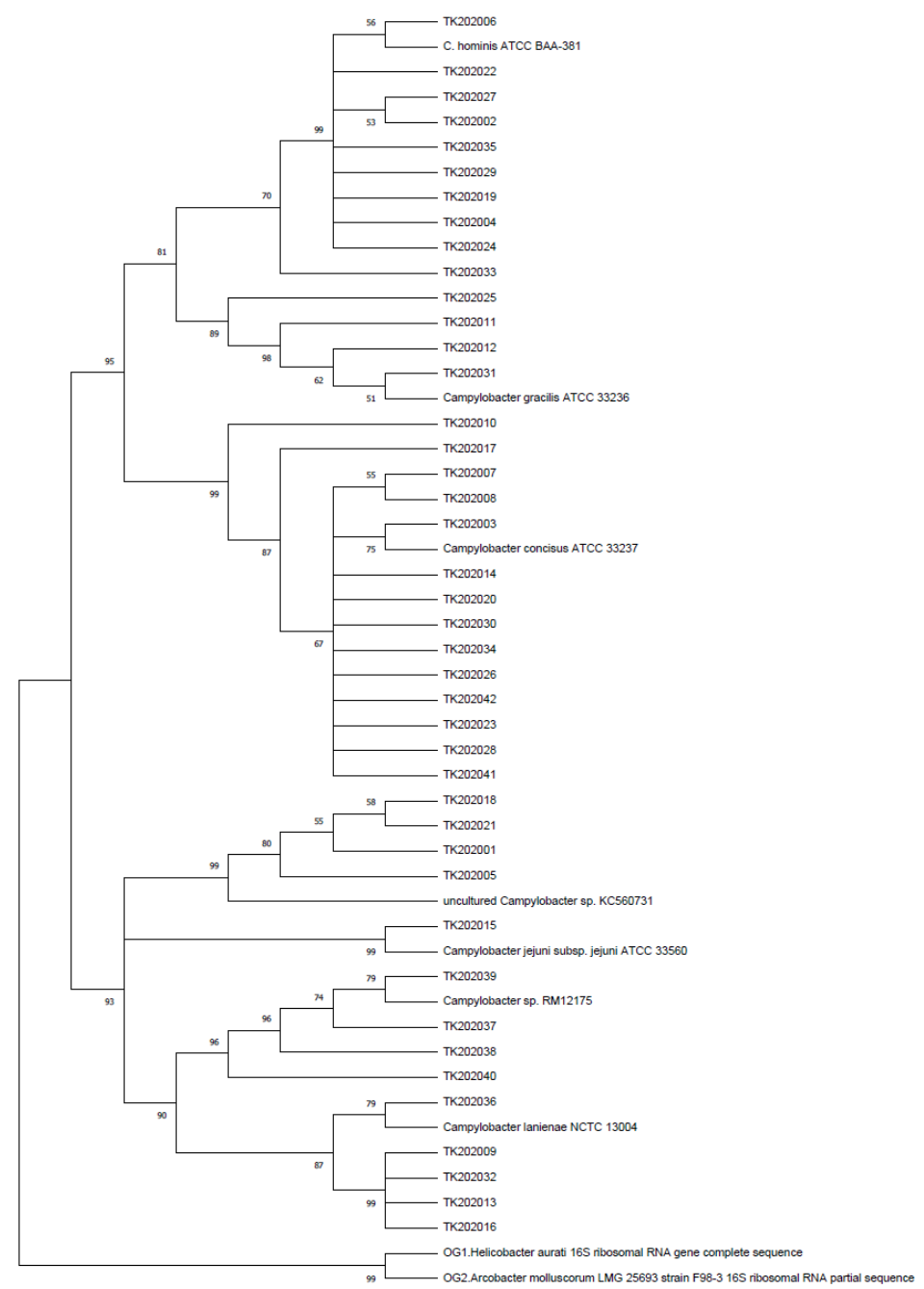

Figure 3. Molecular phylogenetic analysis of Campylobacter species using 16S rRNA sequences by the neighborjoining method. Bootstrap values (\%) based on 1000 replicates are indicated at nodes. The evolutionary distances were computed using the Jukes-Cantor method and are in the units of the number of base substitutions per site. Evolutionary analyses were conducted in MEGA X. Reference strains were included in the analysis. The tree was rooted using $H$. aurati and A. molluscorum

\section{Discussion}

The rapid, accurate isolation and characterization of food-borne pathogens can contribute to the prevention of infectious diseases and save lives by allowing early initiation of effective treatment (Vizzini et al., 2019; Thornval and Hoorfar, 2021). Furthermore, molecular characterization of Campylobacter strains isolated from the same or different samples is very important in tracing human infections to potential sources. Molecular detection techniques based on PCR are fast, but require the extraction of genetic materials (DNA or RNA), specific instrumentation, highly trained laboratory personnel, and hence not suitable for rapid and point-of-care analysis (Vizzini et al., 2019). These methods are also criticized for not being able to differentiate between leaving and dead cells (Thornval and Hoorfar, 2021).

For many years $C$. jejuni and $C$. coli have been the most commonly reported species of Campylobacter associated with gastroenteritis in humans (Moore et al., 2005). Nonetheless, advances in molecular diagnostic techniques have proven that isolation by culture contributes to increased detection rates of C. jejuni and C. coli over other species which biases both the outcome of the diagnosis and the relative contribution of other species to Campylobacter infections (Bullman et al., 2012). The routine culture-based methods fail to detect 
over a third of Campylobacter positive samples (Bullman et al., 2012). The scarcity of atypical Campylobacter species reported so far is attributed to the limitations and bias of culture-based methods (Bullman et al., 2012). The low detection of other Campylobacter species has been associated with their fastidious nature like incubation temperatures, atmospheric conditions, length of incubation, nutrient requirements, and differing susceptibilities to antimicrobial agents required for optimal growth (Bojanić et al., 2019). Also, some injured Campylobacter strains are capable of transforming into a viable but non-culturable (VBNC) state upon exposure to adverse conditions such as suboptimal nutrients, oxygen, osmotic pressure, temperature, and light which complicates their detection (Bullman et al., 2012). This would require the use of enrichment media to enable the growth of desired ones over the contaminants which may overgrow Campylobacter. Therefore, this study reports the detection of Campylobacter spp. in both humans and cattle by molecular methods. To the best of our knowledge, there are limited reports in Tanzania detecting the presence of Campylobacter species directly from faeces without a culturebased approach. Previous authors adopted cultural methods (Komba et al., 2015; Chuma et al., 2016), which, probably, did not give a true picture of Campylobacter-related infections because some of the fastidious species may have failed to grow on selective media.

The reported detection rate of Campylobacter spp. $(65.7 \%)$ in humans was similar to that reported in Nigeria (Gwimi et al., 2015) but higher than those reported previously in Tanzania (Jacob et al., 2011; Chuma et al., 2016), Fiji (Devi et al., 2014), India (Salim et al., 2014) and Cambodia (Osbjer et al., 2016). These observations, probably indicate that the true incidence of non-C. jejuni/C. coli species is probably underestimated as one of the major contributors to human gastroenteritis where routine detection of Campylobacter by culture methods is still used for diagnosis (Bullman et al., 2012). The difference in detection rates could be attributed to the sampling strategy, isolation methods, PCR conditions, level of sanitation, and geographically related variations. Campylobacter concisus and $C$. hominis were the most predominant species occurring at $37.8 \%$ and $21.6 \%$, respectively. This concurs with the findings reported in Denmark (Aabenhus et al., 2002) and Australia (Mahendran et al., 2011). In South Africa, It was previously reported that C. concisus was the second species with a higher prevalence after C. jejuni (Lastovica, 2016). However, C. concisus has been isolated from diarrheic patients without other pathogenic microorganisms suggesting that it could be an emerging cause of human gastroenteritis (Mahendran et al., 2011; Lastovica, 2016). Campylobacter concisus has been reported to be an oral bacterium causing gingivitis and periodontitis but also playing a role in the development of Crohn's disease (CD), a special form of inflammatory bowel disease (IBD) in humans (Liu et al., 2018).

Campylobacter lanienae was detected in both human stool and cattle faecal samples. It has previously been recovered from healthy pigs and cattle (Guévremont et al., 2008). This species has been reported as a probable aetiological agent of human gastroenteritis (Lévesque et al., 2016). However, other workers have suggested that C. lanienae has restricted pathogenicity or is a nonpathogenic Campylobacter (Costa and Iraola, 2019). Campylobacter lanienae was reported to be closely related to the $C$. fetus group but with two special features : (i) additional four to 10 flagellin genes and (ii) the lack of genes necessary for selenium metabolism (Miller et al., 2017). The detection rate of this species was higher than the one previously reported (Inglis and Kalischuk, 2003). Further characterization of C. lanienae could shed more light on its genetic diversity and source (Guévremont et al., 2008). The limited literature on C. lanienae does not allow us to critically assess its epidemiology and the reasons behind its infrequent detection.

Data on concurrent isolation of Campylobacter species in both humans and cattle are limited. In this study, the detection rates were $65.7 \%$ and $20 \%$, in humans and cattle, respectively. Our findings showed higher detection rates when compared to the rates reported earlier in Tanzania (Kusiluka et al., 2005), and Cambodia (Osbjer et al., 2016). However, previous studies started by culturing the faeces which may justify the low detection rates. Further comparative studies on Campylobacter species isolated from humans and cattle are necessary to understand 
their epidemiology and be able to explain the source of infection. However, specific molecular techniques like the whole-genome sequencing (WGS) and multilocus sequence typing (MLST) would be appropriate for source attribution studies.

Since the development of the polymerase chain reaction (PCR) and DNA sequencing, comparison of the gene sequences of various bacterial species has shown that the $16 \mathrm{~S}$ ribosomal RNA gene is highly conserved within a species and among species of the same genus and thus, it can be used as the gold standard for speciation of bacteria (Woo et al., 2002). Phylogenetic analysis based on the 16S rRNA gene is of paramount importance for bacterial taxonomy (Dewhirst et al., 2005) and it has been applied to Brucella (Ntirandekura et al., 2020) and Campylobacter identification (Gorkiewicz et al., 2003). Our findings concur with the reported species of non-C. jejuni/C. coli group with C. concisus being the predominant species (Mukhopadhya et al., 2011). In cattle, our results are in agreement with previously reported occurrences where $C$. lanienae had higher proportions compared to $C$. jejuni and C. hyointestinalis (Guévremont et al., 2008). Figure 3 highlights the taxonomic position of the strains obtained in this study compared to reference strains and supported the sequencing results.

The current study had some limitations including the sample size and lack of culture-based species identification. The sample size used could not allow us to estimate the prevalence or generalize the findings at national or regional levels. This could affect also the diversity of identified Campylobacter strains. Considering that we did not culture the stool samples, the comparison is made based on previous studies carried in sometimes different conditions or settings. Another challenge was the inability to check antimicrobial resistance genes in extracted DNA due to the presence of various DNA from faecal microbiota. However, this study highlights the advantages of molecular methods over culturebased ones in the detection of Campylobacter spp. in clinical samples. We recommend further studies on the burden of disease due to emerging Campylobacter species and associated sequelae necessary for informing policymakers and guide in designing appropriate public health interventions.

\section{Conclusion}

The findings of this study highlight the higher detection rates of the less-frequently isolated Campylobacter species (C. concisus and C. hominis) in patients attending Kilosa District Hospital. Campylobacter lanienae was mainly detected in cattle faecal samples suggesting cattle as another possible reservoir. These Campylobacter species are often neglected due to their cultural behavior and fastidious nature but, they have proven to be zoonotic with a public health concern. It is therefore, important that health practitioners and public health authorities recognize the possibilities of occurrence of Campylobacter species other than C. jejuni and C. coli which are not screened on a routine basis in many countries and hence, go unreported. Molecular-based techniques offer an alternative to culture-based methods especially when it comes to the atypical Campylobacter species as they provide results in a short time and up to species level.

\section{Acknowledgment}

This work was financially supported by the Ministry of Oceans and Fisheries, Korea (grant number 20170488) and the Partnership for Skills in Applied Sciences, Engineering and Technology-Regional Scholarship and Innovation Fund (PASET-RSIF) in collaboration with the Government of the Republic of Korea.

We acknowledge the SACIDS Foundation for One Health for the training and other support provided towards the completion of this study. 
References

Aabenhus, R., Permin, H., On, S. L. W., and Andersen, L. P. (2002). Prevalence of Campylobacter concisus in Diarrhoea of Immunocompromised Patients. Scandinavian Journal of Infectious Diseases, 34(4), 248-252.

ttps://doi.org/10.1080/0036554011008056 6

Al-Nasrawi, H. A. H. A. (2016). Phylogenetic analysis of Campylobacter jejuni from human and birds sources in Iraq. African Journal of Microbiology Research, 10(21), 752-758. https://doi.org/10.5897/AJMR20 15.7890

Amjad, M. (2020). An Overview of the Molecular Methods in the Diagnosis of Gastrointestinal Infectious Diseases [Review Article]. International Journal of Microbiology; Hindawi. https://doi.org/10.1155/2020/81 35724

Berg, R. D. (1996). The indigenous gastrointestinal microflora. Trends in Microbiology, 4(11), 430-435. https://doi.org/10.1016/0966842X(96)10057-3

Bessede, E., Delcamp, A., Sifre, E., Buissonniere, A., and Megraud, F. (2011). New methods for detection of campylobacters in stool samples in comparison to culture. Journal of Clinical Microbiology, 49, 941-944. https://doi.org/10.1128/JCM.014 89-10

Bojanić, K., Midwinter, A. C., Marshall, J. C., Biggs, P. J., and Acke, E. (2019). Isolation of emerging Campylobacter species in working farm dogs and their frozen homekilled raw meat diets. Journal of Veterinary Diagnostic Investigation,
31(1),

23-32.

https://doi.org/10.1177/10406387 18820082

Bronowski, C., James, C. E., and Winstanley, C. (2014). Role of environmental survival in transmission of Campylobacter jejuni. FEMS Microbiology Letters, 356(1), 8-19. https://doi.org/10.1111/1574-6968.12488

Bullman, S., O'Leary, J., Corcoran, D., Sleator, R. D., and Lucey, B. (2012). Molecular-based detection of non-culturable and emerging campylobacteria in patients presenting with gastroenteritis. Epidemiology and Infection, 140, 684-688. https://doi.org/10.1017/S09502688110008 59

Chuma, I. S., Nonga, H. E., Mdegela, R. H., and Kazwala, Rudovick. R. (2016). Epidemiology and RAPD-PCR typing of thermophilic campylobacters from children under five years and chickens in Morogoro Municipality, Tanzania. BMC Infectious Diseases, 16, 1-11.

Coker, A. O., Isokpehi, R. D., Thomas, B. N., Amisu, K. O., and Obi, C. L. (2002). Human campylobacteriosis in developing countries. Emerging Infectious Diseases, 8, 237-243.

Costa, D., and Iraola, G. (2019). Pathogenomics of Emerging Campylobacter Species. Clinical Microbiology Reviews, 32(4). https://doi.org/10.1128/CMR.00072-18

Devi, A., Wilkinson, J., Mahony, T., and Vanniasinkam, T. (2014). Detection of Campylobacter in human faecal samples in Fiji. Journal of Western Pacific Surveillance and Response, 5(4), 30-33. https://doi.org/10.5365/WPSAR.2014.5.2. 007

Dewhirst, F. E., Shen, Z., Scimeca, M. S., Stokes, L. N., Boumenna, T., Chen, T., Paster, B. J., and Fox, J. G. (2005). Discordant 165 and $23 S$ rRNA gene phylogenies for the genus Helicobacter: Implications for phylogenetic inference and systematics. Journal of Bacteriology, 187, 6106-6118. 
https:// doi.org/10.1128/JB.187.17.6106-

6118.2005

Edgar, R. C. (2004). MUSCLE: Multiple sequence alignment with high accuracy and high throughput. Nucleic Acids Research, 32, 17921797.

https://doi.org/10.1093/nar/gk h340

Esan, O. B., Pearce, M., van Hecke, O., Roberts, N., Collins, D. R. J., Violato, M., McCarthy, N., Perera, R., and Fanshawe, T. R. (2017). Factors Associated with Sequelae of Campylobacter and Nontyphoidal Salmonella Infections: A Systematic Review. Ebiomedicine, 15, 100-111. https://doi.org/10.1016/j.ebiom.2 016.12.006

Felsenstein, J. (1985). Confidence limits on phylogenies: An approach using the bootstrap. Evolution, 39, 783791.

https://doi.org/10.1111/j.15585646.1985.tb00420.x

Gahamanyi, N., Mboera, L. E. G., Matee, M. I., Mutangana, D., and Komba, E. V. G. (2020, January 14). Prevalence, risk factors, and antimicrobial resistance Profiles of thermophilic Campylobacter species in humans and animals in Sub-Saharan Africa: A systematic review [Review Article]. International Journal of Microbiology; Hindawi. https://doi.org/10.1155/2020/20 92478

Gahamanyi, N., Song, D.-G., Yoon, K.-Y., Mboera, L. E. G., Matee, M. I., Mutangana, D., Komba, E. V. G., Pan, C.-H., and Amachawadi, R. G. (2021). Genomic Characterization of Fluoroquinolone-Resistant Thermophilic Campylobacter Strains Isolated from Layer Chicken Feces in Gangneung, South Korea by Whole-Genome
Sequencing. Genes, 12(8), 1131. https://doi.org/10.3390/genes120 81131

Ghosh, R., Uppal, B., Aggarwal, P., Chakravarti, A., Jha, A. K., and Dubey, A. P. (2014). A Comparative Study of Conventional and Molecular Techniques in Diagnosis of Campylobacter Gastroenteritis in

Children. Annals of Clinical and Laboratory Science, 44(1), 42-48.

Gorkiewicz, G., Feierl, G., Schober, C., Dieber, F., Köfer, J., Zechner, R., and Zechner, E. L. (2003). Species-specific identification of campylobacters by partial $16 \mathrm{~S}$ rRNA gene sequencing. Journal of Clinical Microbiology, 41 , 2537-2546. https://doi.org/10.1128/JCM.41.6.25372546.2003

Guévremont, E., Nadeau, É., Sirois, M., and Quessy, S. (2006). Antimicrobial susceptibilities of thermophilic Campylobacter from humans, swine, and chicken broilers. Canadian Journal of Veterinary Research, 70(2), 81-86.

Guévremont, E., Normand, V., Lamoureux, L., and Côté, C. (2008). Genetic detection of Campylobacter lanienae in fecal matter and stored manure from swine and dairy cattle. Foodborne Pathogens and Disease, 5, 361-364. https://doi.org/10.1089/fpd.2007.0054

Gwimi, P. B., Faleke, O. O., Salihu, M. D., Magaji, A. A., Abubakar, M. B., Nwankwo, I. O., and Ibitoye, E. B. (2015). Prevalence of Campylobacter species in fecal samples of pigs and humans from Zuru Kebbi State, Nigeria. International Journal of One Health, $1,1-5$.

Hall, T. A. (1999). BioEdit: A user-friendly biological sequence alignment editor and analysis program for Windows 95/98/NT. In Nucleic Acids Symposium Series, 41(41), 95-98.

Havelaar, A. H., Ivarsson, S., Löfdahl, M., and Nauta, M. J. (2013). Estimating the true incidence of campylobacteriosis and 
salmonellosis in the European Union, 2009. Epidemiology and Infection, 141(2), 293-302. https://doi.org/10.1017/S09502688120005 68

Inglis, G. D., and Kalischuk, L. D. (2003). Use of PCR for Direct Detection of Campylobacter Species in Bovine Feces. Applied and Environmental Microbiology, 69(6), 34353447. https://doi.org/10.1128/AEM.69.6.34353447.2003

Jacob, P., Mdegela, R. H., and Nonga, H. E. (2011). Comparison of Cape Town and Skirrow's Campylobacter isolation protocols in humans and broilers in Morogoro, Tanzania. Tropical Animal Health and Production, 43, 1007-1013. https://doi.org/DOI

10.1007/s11250-011-9799-z

Jukes, T. H., and Cantor, C. R. (1969). Evolution of protein molecules. In H. N. Munro (Ed.), Mammalian protein metabolism (21 ${ }^{\text {st }}$ ed., Vol. 13, pp. 21-132). Academic Press, New York.

Kaakoush, N. O., Castaño-Rodríguez, N., Mitchell, H. M., and Man, S. M. (2015). Global Epidemiology of Campylobacter Infection. Clinical Microbiology Reviews, 28(3), 687720.

https://doi.org/10.1128/CMR.00 006-15

Komba, E. V. G., Mdegela, R. H., Msoffe, P. L., and Ingmer, H. (2013). Human and animal Campylobacteriosis in Tanzania: A review. Tanzania Journal of Health Research, 15(1), 113.

https://doi.org/10.4314/thrb.v15i 1.6

Komba, E. V. G., Mdegela, R. H., Msoffe, P. L. M., Nielsen, L. N., and Ingmer, H. (2015). Prevalence, antimicrobial resistance and risk factors for thermophilic Campylobacter infections in symptomatic and asymptomatic humans in Tanzania. Zoonoses and Public Health, 62, 557-568.

Kumar, S., Stecher, G., Li, M., Knyaz, C., and Tamura, K. (2018). MEGA X: Molecular Evolutionary Genetics Analysis across computing platforms. Molecular Biology and Evolution, 35, 1547-1549. https://doi.org/10.1093/molbev/ msy096

Kusiluka, L. J. M., Karimuribo, E. D., Mdegela, R. H., Luoga, E. J., Munishi, P. K. T., Mlozi, M. R. S., and Kambarage, D. M. (2005). Prevalence and impact of water-borne zoonotic pathogens in water, cattle and humans in selected villages in Dodoma Rural and Bagamoyo districts, Tanzania. Physics and Chemistry of the Earth, Parts A/B/C, 30(11-16), 818-825.

Lastovica, A. J. (2006). Emerging Campylobacter spp.: The tip of the iceberg. Clinical Microbiology Newsletter, 28(7), 49-56. https://doi.org/10.1016/j.clinmicnews.20 06.03.004

Lastovica, A. J. (2016). Non-jejuni/ coli Campylobacter Species and Related Organisms in Poultry, and Their Relevance in Human and Animal Disease. In B. B. Fonseca, H. Fernandez, and D. A. Rossi (Eds.), Campylobacter spp. And Related Organisms in Poultry: Pathogen-Host Interactions, Diagnosis and Epidemiology (pp. 185-206). Springer International Publishing. https://doi.org/10.1007/9783-319-29907-5_11

Lévesque, S., Lemay, F., Bekal, S., Frost, E. H., and Michaud, S. (2016). First reported case of Campylobacter lanienae enteritis in a human. JMM Case Reports, 3(3), 1-5. https://doi.org/10.1099/jmmcr.0.005045

Linscott, A. J., Flamholtz, R. B., Shukla, D., Song, Y., Liu, C., and Finegold, S. M. (2005). Fatal septicemia due to Clostridium hathewayi and Campylobacter hominis. Anaerobe, 11, 97-98. https://doi.org/10.1016/j.anaerobe.2004.1 0.002 
Linton, D., Lawson, A. J., Owen, R. J., and Stanley, J. (1997). PCR detection, identification to species level, and fingerprinting of Campylobacter jejuni and Campylobacter coli direct from diarrheic samples. Journal of Clinical Microbiology, 35(10), 2568-2572.

Linton, D., Owen, R. J., and Stanley, J. (1996). Rapid identification by PCR of the genus Campylobacter and of five Campylobacter species enteropathogenic for man and animals. Research in Microbiology, 147, 707718. https://doi.org/10.1016/S09232508(97)85118-2

Liu, F., Ma, R., Wang, Y., and Zhang, L. (2018). The Clinical Importance of Campylobacter concisus and Other Human Hosted Campylobacter Species. Frontiers in Cellular and Infection Microbiology, 8. https://doi.org/10.3389/fcimb.20 18.00243

Mahendran, V., Riordan, S. M., Grimm, M. C., Tran, T. A. T., Major, J., Kaakoush, N. O., Mitchell, H., and Zhang, L. (2011). Prevalence of Campylobacter Species in Adult Crohn's Disease and the Preferential Colonization Sites of Campylobacter Species in the Human Intestine. PLOS ONE, 6(9), e25417.

https:// doi.org/10.1371/journal.p one.0025417

Man, S. M. (2011). The clinical importance of emerging Campylobacter species. Nature Reviews Gastroenterology and Hepatology, 8(12), 669-685. https://doi.org/10.1038/nrgastro. 2011.191

McLain, J. E., Cytryn, E., Durso, L. M., and Young, S. (2016). Culture-based Methods for Detection of Antibiotic Resistance in Agroecosystems: Advantages, Challenges, and Gaps in Knowledge. Journal of Environmental Quality, 45(2), 432440 . https://doi.org/10.2134/jeq2015.0 6.0317

Miller, W. G., Yee, E., Lopes, B. S., Chapman, M. H., Huynh, S., Bono, J. L., Parker, C. T., Strachan, N. J. C., and Forbes, K. J. (2017). Comparative Genomic Analysis Identifies a Campylobacter Clade Deficient in Selenium Metabolism. Genome Biology and Evolution, 9(7), 1843-1858. https://doi.org/10.1093/gbe/evx 093

Moore, J. E., Corcoran, D., Dooley, J. S. G., Fanning, S., Lucey, B., Matsuda, M., McDowell, D. A., Mégraud, F., Millar, B. C., O'Mahony, R., O'Riordan, L., O'Rourke, M., Rao, J. R., Rooney, P. J., Sails, A., and Whyte, P. (2005). Campylobacter. Veterinary Research, 36(3), 351-382. https://doi.org/10.1051/vetres:2005012

Mshelia, G. D., Amin, J. D., Woldehiwet, Z., Murray, R. D., and Egwu, G. O. (2010). Epidemiology of Bovine Venereal Campylobacteriosis: Geographic Distribution and Recent Advances in Molecular Diagnostic Techniques. Reproduction in Domestic Animals, 45(5), e221-e230. https://doi.org/10.1111/j.14390531.2009.01546.x

Mukhopadhya, I., Thomson, J. M., Hansen, R., Berry, S. H., El-Omar, E. M., and Hold, G. L. (2011). Detection of Campylobacter concisus and other Campylobacter Species in colonic biopsies from adults with Ulcerative Colitis. PLOS ONE, 6(6), 1-7. https://doi.org/10.1371/journal.pone.002 1490

Ntirandekura, J. B., Makene, V. A., Kasanga, C. J., Matemba, L. E., Kimera, S. I., Muma, J. B., and Karimuribo, E. D. (2020). Molecular characterization of Brucella species detected in humans and domestic ruminants of pastoral areas in Kagera ecosystem, Tanzania. Veterinary Medicine and Science, $n / a(\mathrm{n} / \mathrm{a})$, $1-8$. https://doi.org/10.1002/vms3.298 
Osbjer, K., Tano, E., Chhayheng, L., Mac-Kwashie, A. O., Fernström, L.-L., Ellström, P., Sokerya, S., Sokheng, C., Mom, V., Chheng, K., San, S., Davun, H., Boqvist, S., Rautelin, H., and Magnusson, U. (2016). Detection of Campylobacter in human and animal field samples in Cambodia. APMIS, 124(6), 508515. https://doi.org/10.1111/apm.12531

Park, S. F. (2002). The physiology of Campylobacter species and its relevance to their role as foodborne pathogens. International Journal of Food Microbiology, 74, 177-188.

Ruiz-Palacios, G. M. (2007). The health burden of Campylobacter infection and the impact of antimicrobial resistance: Playing chicken. Clinical Infectious Diseases: An Official Publication of the Infectious Diseases Society of America, 44, 701-703.

Rukambile, E., Sintchenko, V., Muscatello, G., Wang, Q., Kiiru, J., Maulaga, W., Magidanga, B., Banda, G., Kock, R., and Alders, R. (2021). Campylobacter and Salmonella in Scavenging Indigenous Chickens in Rural Central Tanzania: Prevalence, Antimicrobial Resistance, and Genomic Features. Microbiology Research, 12(2), 440454.

https://doi.org/10.3390/microbio lres12020030

Sahin, O., Yaeger, M., Wu, Z., and Zhang, Q. (2017). Campylobacter-associated diseases in animals. Annual Review of Animal Biosciences, 5, 21-42. https://doi.org/10.1146/annurev -animal-022516-022826

Saitou, N., and Nei, M. (1987). The neighbor-joining method: A new method for reconstructing phylogenetic trees. Molecular Biology and Evolution, 4, 406-425. https:// doi.org/10.1093/oxfordjo urnals.molbev.a040454

Salim, S. M., Mandal, J., and Parija, S. (2014). Isolation of Campylobacter from human stool samples. Indian
Journal of Medical Microbiology, 32(1), 35-38. https://doi.org/10.4103/02550857.124294

Singh, H., Rathore, R. S., Singh, S., and Cheema, P. S. (2011). Comparative analysis of cultural isolation and PCR based assay for detection of Campylobacter jejuni in food and faecal samples. Brazilian Journal of Microbiology, 42, 181-186. https://doi.org/10.1590/S151783822011000100022

Sproston, E. L., Wimalarathna, H. M. L., and Sheppard, S. K. (2018). Trends in fluoroquinolone resistance in Campylobacter. Microbial Genomics, 4(8). https://doi.org/10.1099/mgen.0.000198

Sulaiman, I. M., Hsieh, Y.-H., and Simpson, S. (2020). Species identification of Campylobacter jejuni and Campylobacter coli isolates from raw poultry products by MALDI-TOF MS and rRNA Sequence analysis. Journal of Aoac International, 103(1), 197-204. https://doi.org/10.5740/jaoacint.19-0170

Thornval, N. R., and Hoorfar, J. (2021). Progress in detection of Campylobacter in the food production chain. Current Opinion in Food Science, 39, 16-21. https://doi.org/10.1016/j.cofs.2020.12.001

Vizzini, P., Braidot, M., Vidic, J., and Manzano, M. (2019). Electrochemical and Optical Biosensors for the Detection of Campylobacter and Listeria: An Update Look. Micromachines, 10(8), 500. https://doi.org/10.3390/mi10080500

Woo, P. C. Y., Leung, K. W., Tsoi, H. W., Wong, S. S. Y., Teng, J. L. L., and Yuen, K. Y. (2002). Thermo-tolerant Campylobacter fetus bacteraemia identified by $16 \mathrm{~S}$ ribosomal RNA gene sequencing: An emerging pathogen in immunocompromised patients. Journal of Medical Microbiology, 51(9), 740-746. https://doi.org/10.1099/0022-1317-51-9740 
Yamazaki-Matsune, W., Taguchi, M., Seto, K., Kawahara, R., Kawatsu, K., Kumeda, Y., Kitazato, M., Nukina, M., Misawa, N., and Tsukamoto, T. (2007). Development of a multiplex PCR assay for identification of Campylobacter coli, Campylobacter fetus, Campylobacter hyointestinalis subsp. hyointestinalis, Campylobacter jejuni, Campylobacter lari and Campylobacter upsaliensis. Journal of Medical Microbiology, 56(11), 1467-1473.
Zhang, L., Man, S. M., Day, A. S., Leach, S. T., Lemberg, D. A., Dutt, S., Stormon, M., Otley, A., O'Loughlin, E. V., Magoffin, A., Ng, P. H. Y., and Mitchell, H. (2009). Detection and Isolation of Campylobacter Species Other than C. jejuni from Children with Crohn's Disease. Journal of Clinical Microbiology, 47(2), 453-455. https://doi.org/10.1128/JCM.01949-08 